\title{
Competitive channels in the photodissociation of thionyl chloride
}

\author{
Melanie Roth, Christof Maul* and Karl-Heinz Gericke \\ Institut für Physikalische und Theoretische Chemie, Technische Universität Braunschweig, \\ Hans-Sommer-Str. 10, 38106, Braunschweig, Germany.E-mail: c.maul@tu-braunschweig.de
}

Received 2nd January 2002, Accepted 8th April 2002

First published as an Advance Article on the web 30th May 2002

\begin{abstract}
The photodissociation dynamics of thionyl chloride are investigated by a one-dimensional resonance enhanced multi-photon/time-of-flight technique (REMPI/TOF) at a dissociation wavelength around $235 \mathrm{~nm}$. Nascent sulfur monoxide molecules and chlorine atoms were detected state-specifically under collision-free conditions for parallel and perpendicular polarisation geometries. Dissociation and detection were performed using the same laser. Polarisation-dependent and state-specific TOF profiles were converted into kinetic energy distributions using a least squares fitting method taking into account velocity-dependent spatial fragment anisotropies. Kinetic energy distributions are narrow and structureless for SO and bimodal for both spin-orbit states of $\mathrm{Cl}$. The bimodality reflects the competition between two- and three-body decay: $\mathrm{SOCl}_{2}+h \nu \rightarrow$ $\mathrm{SOCl}+\mathrm{Cl}$ and $\mathrm{SOCl}_{2}+h \nu \rightarrow \mathrm{SO}+2 \mathrm{Cl}$. Chlorine atoms are preferentially released along the polarisation vector of the dissociation laser. The spatial distribution of $\mathrm{Cl}$ is characterised by an anisotropy parameter of $0.8 \pm 0.2$ for the two-body decay and $0.2 \pm 0.2$ for the three-body decay. SO molecules are isotropically released with low kinetic energy together with two ground state chlorine atoms. These products are most likely to be generated in a sequential three-body decay on a potential energy surface of $\mathrm{A}^{\prime}$ symmetry. Another surface of $\mathrm{A}^{\prime \prime}$ symmetry instantaneously produces fast chlorine atoms in a two-body decay process to equal amounts in the ground and the excited spin-orbit states. The corresponding $\mathrm{SOCl}$ fragment carries about $50 \%$ of the available energy as internal energy.
\end{abstract}

\section{A. Introduction}

Quantum state resolved photoinduced three-body decay on a state-resolved level is a scarcely investigated elementary process in physical chemistry, although three-body processes are crucial for many complex reaction schemes in atmospheric and combustion chemistry. Due to refined experimental and theoretical techniques, ${ }^{1,2}$ interest in three-body decay processes has increased markedly in the past few years, and only recently the topic has been extensively reviewed. ${ }^{3-5}$

Carbonyl and thionyl compounds play a major role in the investigation of three-body decay processes for two reasons. First, their dissociation energy with respect to fragmentation into three products is comparatively low, i.e. generally below $50000 \mathrm{~cm}^{-1}$, giving rise to easily accessible absorption bands in a spectral regime with wavelengths greater than $200 \mathrm{~nm}$. Second, SO and CO products can easily be detected in a state-selective and energy-sensitive method e.g. the REMPI/ TOF technique. If the remaining constituents of the compound are chosen such that they are likewise detectable, e.g. $\mathrm{Cl}, \mathrm{Br}$, or $\mathrm{CH}_{3}$, the most complete characterization of internal and kinetic fragment energies, which can be achieved by non-coincidence methods, becomes possible. Therefore, a number of carbonyl and thionyl compounds, namely acetone, ${ }^{6-9}$ various acetyl halides, ${ }^{10-14}$ phosgene,${ }^{15-17}$ carbonyl chloride fluoride, ${ }^{18}$ dimethyl sulfoxide, ${ }^{19}$ and thionyl chloride,${ }^{20-25}$ have been the subjects of three-body decay studies.

Thionyl chloride is a non-planar molecule which belongs to the $C_{\mathrm{s}}$ point group, with a ${ }^{1} \mathrm{~A}^{\prime}$ ground state. It is of particular interest for three-body decay studies because its absorption spectrum, as shown in Fig. 1, extends well into the spectral range easily accessible with ultraviolet laser radiation. ${ }^{26}$ The distinct shoulder at $40000 \mathrm{~cm}^{-1}(250 \mathrm{~nm})$ and the pronounced absorption maximum above $50000 \mathrm{~cm}^{-1}$ (below $200 \mathrm{~nm}$ ) were assigned to overlapping electronic transitions: the $\sigma^{*}{ }_{\mathrm{SCl}} \leftarrow \mathrm{n}_{\mathrm{S}}$ and the $\pi^{*}$ so $\leftarrow \mathrm{n}_{\mathrm{S}}$ transitions from a non-bonding lone pair electron on the sulfur atom to the anti-bonding molecular orbitals of the $\mathrm{S}-\mathrm{Cl}$ or the $\mathrm{S}-\mathrm{O}$ bond, and, at higher excitation energies, the corresponding transitions from a non-bonding $\mathrm{n}_{\mathrm{Cl}}$ electron out of the $\mathrm{Cl}(3 \mathrm{p})$ manifold..$^{24,27}$

Photodissociation studies have been performed at wavelengths of $193 \mathrm{~nm}, 235 \mathrm{~nm}$, and $248 \mathrm{~nm},{ }^{20-24,28}$ corresponding to excitation in the short wavelength absorption maximum and the long wavelength shoulder, respectively. The dissociation dynamics differ remarkably for dissociation at $193 \mathrm{~nm}$ and at the longer wavelengths. At $193 \mathrm{~nm}$ the main dissociation channel has been found to be a concerted three-body decay

$$
\mathrm{SOCl}_{2}+h \nu \rightarrow \mathrm{SO}+2 \mathrm{Cl}, \Delta H(1)=37950 \pm 100 \mathrm{~cm}^{-1}
$$

accounting for more than $80 \%$ of the overall process, accompanied by a radical channel $(17 \%)$

$$
\mathrm{SOCl}_{2}+h \nu \rightarrow \mathrm{SOCl}+\mathrm{Cl}, \Delta H(2)=20050 \pm 100 \mathrm{~cm}^{-1}
$$

and a molecular channel $(\leqslant 3 \%)^{22,23}$

$$
\mathrm{SOCl}_{2}+h \nu \rightarrow \mathrm{SO}+\mathrm{Cl}_{2}, \Delta H(3)=17950 \pm 100 \mathrm{~cm}^{-1}
$$

Dissociation enthalpies $\Delta H$ are given for $0 \mathrm{~K}^{22}$ At $248 \mathrm{~nm}$ no contribution of the three-body channel (1) has been observed, whereas the radical channel (2) becomes the main channel $(96.5 \%)$, and the molecular channel (3) again contributes only insignificantly $(3.5 \%)$ to the dissociation process. $^{22,23}$ These results were obtained by photofragment translational spectroscopy (PTS), which is capable of completely monitoring all fragments, but is insensitive to product quantum states due to the utilization of electron bombardment for fragment ionisation. The data agree remarkably well with the results from the state-selective observation of SO fragments 


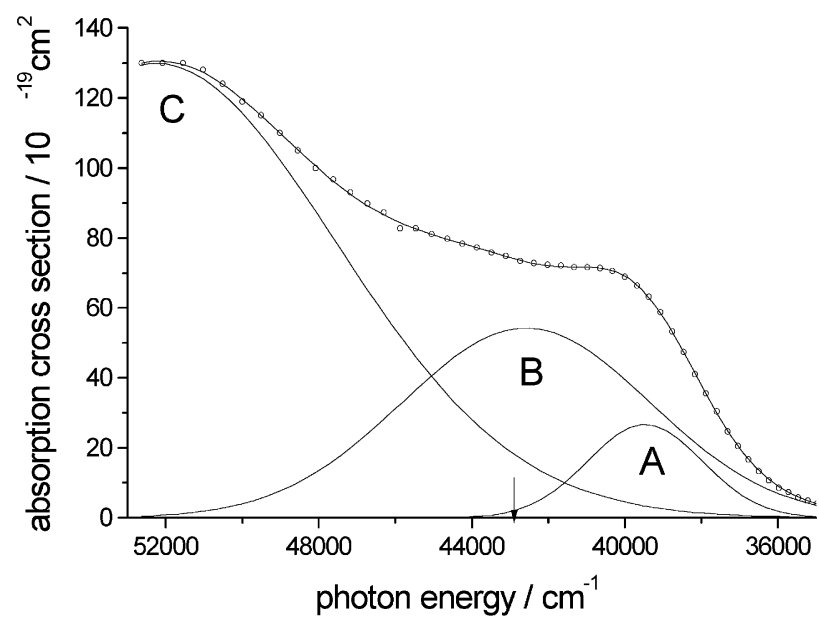

Fig. 1 Absorption spectrum of $\mathrm{SOCl}_{2}$. Data are taken from ref. 26 . The spectrum can be fitted by three Gaussians centered at $39500 \mathrm{~cm}^{-1}$ $(253 \mathrm{~nm}), 42500 \mathrm{~cm}^{-1}(235 \mathrm{~nm})$, and $52500 \mathrm{~cm}^{-1}(191 \mathrm{~nm})$, respectively. The first maximum (A) at $39500 \mathrm{~cm}^{-1}$ has been assigned to a $\pi^{*}$ SO $\leftarrow \mathrm{n}_{\mathrm{S}}$ transition from a non-bonding lone pair electron on the sulfur atom to the anti-bonding $\pi^{*}$ molecular orbital of the $\mathrm{S}-\mathrm{O}$ bond. The second (B) and third (C) maxima at $42500 \mathrm{~cm}^{-1}$ and at 52500 $\mathrm{cm}^{-1}$ have been considered to result from $\mathrm{n}_{\mathrm{S}}$ or $\mathrm{n}_{\mathrm{Cl}}$ excitation to the anti-bonding $\sigma^{*} \mathrm{SCl}$ orbitals. Selecting the dissociation wavelength at $235 \mathrm{~nm}$ should result in competing dissociation mechanisms originating from the respective excited states $\mathrm{B}$ and $\mathrm{C}$.

at both dissociation wavelengths employing laser induced fluorescence (LIF) ${ }^{20,21}$ and microwave spectroscopy. ${ }^{29}$ At 235 $\mathrm{nm}$, bimodal kinetic energy distributions with a large contribution of slow ground state $\left.\mathrm{Cl}^{2}{ }^{2} \mathrm{P}_{3 / 2}\right)$ fragments observed in a state-selective photofragment imaging experiment hinted at the onset of three-body decay in competition with the radical channel. ${ }^{28}$

In a continuation of previous experiments, a more comprehensive study of this three-body prototype reaction is desirable. Therefore, first we chose to utilize REMPI/TOF in order to obtain information about quantum state populations for both $\mathrm{SO}$ and $\mathrm{Cl}$ fragments. Second, we selected the dissociation wavelength of $235 \mathrm{~nm}$, in order to investigate the competition between three-body and radical channels for identical energy conditions.

\section{B. Experimental}

A detailed description of the experimental set-up has been given elsewhere. ${ }^{30}$ It consists of a home-built single-field time-of-flight (TOF) spectrometer with a length ratio of the acceleration to the drift region of 1:2 at a total length of 57 $\mathrm{cm}$. The spectrometer was evacuated to a base pressure of $10^{-4} \mathrm{~Pa}\left(10^{-6} \mathrm{mbar}\right)$ by a $3601 \mathrm{~s}^{-1}$ turbo molecular pump and two $500 \mathrm{l} \mathrm{s}^{-1}$ oil diffusion pumps (Leybold Baur 1). Thionyl chloride was mixed with helium in a ratio of $c a$. 1:25 and was fed into the spectrometer via a supersonic jet, generated in an inductively driven pulsed nozzle (General Valve Series 9). The nozzle diameter was $0.5 \mathrm{~mm}$, and the valve was operated at a stagnation pressure of typically $5 \times 10^{4} \mathrm{~Pa}(500 \mathrm{mbar})$ and a pulse duration of $250 \mu \mathrm{s}$, resulting in an operational background pressure of less than $2 \times 10^{-3} \mathrm{~Pa}\left(2 \times 10^{-5} \mathrm{mbar}\right)$ at a repetition rate of $10 \mathrm{~Hz}$. The temperature in the supersonic beam was $13 \mathrm{~K}$, determined from the rotational distribution of $\mathrm{CO}$ molecules under similar expansion conditions. ${ }^{15}$ The gas mixture had to be renewed frequently in order to maintain stable experimental conditions, because $\mathrm{SOCl}_{2}$ hydrolysis takes place in the gas lines, which can conveniently be monitored by a decrease in the $\mathrm{SOCl}_{2}$ signal accompanied by a corresponding increase in the $\mathrm{HCl}$ signal.
Simultaneous dissociation of thionyl chloride and stateselective detection of $\mathrm{SO}$ molecules or $\mathrm{Cl}$ atoms was performed using an excimer laser pumped dye laser (Lambda Physik LPD 3000, Lambda Physik LPX 100) operated with Coumarin-47. The dye laser repetition rate was chosen to be $5 \mathrm{~Hz}$ in order to eliminate the shot-to-shot time jitter imposed onto the TOF signal by the expansion characteristics of the supersonic valve, which differ in a systematic way for two consecutive gas pulses. The dye laser output was frequency doubled by a BBO crystal and focused into the spectrometer by an $80 \mathrm{~mm}$ lens. The laser beam intersected the molecular beam at an angle of $54^{\circ}$, while the spectrometer formed an angle of $90^{\circ}$ with the propagation directions of both the laser and the molecular beam. The angle between the spectrometer axis and the electric field vector of the linearly polarised laser light could be varied from $0^{\circ}$ to $90^{\circ}$ in order to investigate the spatial fragment distribution. The intensity of the laser light and the particle density in the supersonic beam were carefully controlled to avoid kinetic energy transfer onto the fragments due to space charge effects. The laser intensity was typically kept below $500 \mu \mathrm{J}$ per pulse.

The $\mathrm{SO}\left(\mathrm{X}^{3} \Sigma^{-}\right)$fragments were ionised around $235.5 \mathrm{~nm}$ by $(1+1)$-REMPI, using the $\mathrm{d}{ }^{1} \Pi$ state as resonant intermediate. ${ }^{31}$ From the ionisation potential of $82994 \mathrm{~cm}^{-1}$, a threshold of $241 \mathrm{~nm}$ for $(1+1)$-excitation is calculated. ${ }^{32}$ Atomic chlorine was resonantly ionised by a $(2+1)$-REMPI scheme, employing the $4 p^{2} \mathrm{D}_{3 / 2} \leftarrow 3 \mathrm{p}^{2} \mathrm{P}_{3 / 2}$ transition at $235.336 \mathrm{~nm}$ for ground state atoms and the $4 \mathrm{p}^{2} \mathrm{P}_{1 / 2} \leftarrow 3 \mathrm{p}^{2} \mathrm{P}_{1 / 2}$ transition at $235.205 \mathrm{~nm}$ for excited spin-orbit state atoms as resonance enhanced steps. $^{33,34}$

Ions were detected by a double stage multi-channel plate assembly (Galileo) with an active diameter of $40 \mathrm{~mm}$. In the spectrometer the ions were accelerated by a moderate static electric field of typically $10^{3} \mathrm{~V} \mathrm{~m}^{-1}$ in the acceleration region and detected after passing the drift tube. Here the ions were mass selected, and instead of the total speed $v$, the measured quantity was the velocity component $v_{z}$ along the spectrometer axis, monitored by the mass peak broadening. A linear relationship between the velocity component $v_{z}$ and the deviation $t-t_{0}$ from the centre $t_{0}$ of the time of flight profile holds for our spectrometer geometry:

$$
t-t_{0}=\frac{3 t_{0}^{2}}{8 s} v_{z}
$$

where $s=0.57 \mathrm{~m}$ is the total length of the spectrometer, consisting of a $19 \mathrm{~cm}$ long acceleration region and a $38 \mathrm{~cm}$ long drift region. Experimental parameters like the acceleration voltage, the ion mass, and the ion charge are contained in the (experimentally observable) quantity $t_{0}$. Since in our experimental set-up the velocity component $v_{z}$ is perpendicular to the velocity of the molecular beam, no laboratory to centre of mass transformation has to be performed. The ion signal is monitored by a digital oscilloscope (LeCroy 9450). Ion flyout which occurs if the transversal velocity component of the ions is larger than the ratio of the detector radius to the time of flight is accounted for in the analysis procedure, as is the case for a biased detection of fragments that occurs if the Doppler broadening of the transition exceeds the laser linewidth.

If strong acceleration fields of the order of $10^{4} \mathrm{~V} \mathrm{~m}^{-1}$ are applied, ion fly-out is suppressed and the arrival at the particle detector is assured for all ions generated in one laser pulse. This mode mainly serves for the determination of quantum state populations. Additionally, although the Doppler broadening of the spectral lines has not been used for obtaining kinetic energy distributions from the line shape due to the much lower accuracy compared to the evaluation of the TOF data, it is a most welcome tool to check for the correctness of the kinetic energy distributions, derived from the TOF measurements, by an independent method. 
The accuracy of the set-up for determining kinetic energies was determined by the well-characterised photodissociation of molecular $\mathrm{Cl}_{2}$ at $308 \mathrm{~nm} .^{32,35,36}$ The experimentally determined values are in excellent agreement with the values that were calculated from the spectrometer geometry. The width (FWHM) of the response function of the spectrometer is mainly determined by the laser pulse duration and amounts to $c a$. $10 \mathrm{~ns}$. The kinetic energy resolution is energy dependent and is approximately $5 \%$ for the energy values encountered here. Immediately after each measurement the background signal was monitored with the laser delayed with respect to the gas pulse under otherwise identical conditions and subtracted from the previously obtained TOF profile. Further data processing was performed by a personal computer.

\section{Results}

Since the dissociation channels (1) through (3) discussed in the Introduction produce chemically different product species, a comprehensive characterisation of the $\mathrm{SOCl}_{2}$ fragmentation requires the detection of more than just one dissociation product. Because of the ease of detection, the molecular fragment $\mathrm{SO}$ and the atomic fragment $\mathrm{Cl}$ were chosen as the fragments to be monitored by the state and energy sensitive REMPI/ TOF detection technique. Above all, several detection wavelengths for both species are found around $235 \mathrm{~nm}$ where the parent molecule $\mathrm{SOCl}_{2}$ exhibits a conveniently large absorption cross section, presumably leading to a simultaneous excitation of more than one potential energy surface. Therefore, one-colour dissociation experiments can be performed in order to investigate competing fragmentation channels by monitoring both fragments, without inducing artefacts associated with a change in the excitation energy upon changing the detection wavelength. Neither $\mathrm{SOCl}$ nor $\mathrm{Cl}_{2}$ are accessible to a similar detection scheme.

\section{Sulfur monoxide}

Sulfur monoxide can be produced from $\mathrm{SOCl}_{2}$ photodissociation either via a three-body decay (1) or via the molecular channel (3). The corresponding available energies are calculated as the difference of the photon energy $h \nu=42450 \pm 50$ $\mathrm{cm}^{-1}$ and the dissociation enthalpies $\Delta H$ from eqn. (1) and (3). The uncertainty of $50 \mathrm{~cm}^{-1}$ reflects the energy variation when tuning the analysis laser. Available energies of $4500 \pm 150 \mathrm{~cm}^{-1}$ and $24500 \pm 150 \mathrm{~cm}^{-1}$ are obtained for ground state fragments from three-body decay (1) and molecular channel (3), respectively. Therefore, both channels can easily be discriminated from each other in terms of energy partitioning onto the SO fragment. The upper trace of Fig. 2 shows a rotationally resolved spectrum of the SO fragment obtained for the one-colour dissociation between $235.2 \mathrm{~nm}$ and $235.8 \mathrm{~nm}$. This transition has only recently been observed for the first time, ${ }^{37}$ with the assignment of the excited state remaining subject to discussion. ${ }^{31,38}$ The authors agree, however, that the transition originates from the $v^{\prime \prime}=1$ manifold of the $\mathrm{SO}\left(\mathrm{X}^{3} \Sigma^{-}\right)$electronic ground state. Here, this band was chosen in order to match the dissociation wavelengths when monitoring SO to the conditions when monitoring $\mathrm{Cl}$. In view of the small rotational constant of ground state SO (see Table 1), the pronounced bandhead structure and the relatively short rotational progression indicate only a small rotational excitation of the SO fragment.

Various rotationally resolved lines have been used to obtain state-resolved time-of-flight profiles. In Fig. 3 a typical TOF profile is shown for an excitation wavelength of $235.756 \mathrm{~nm}$ for both perpendicular $\left(90^{\circ}\right)$ and parallel $\left(0^{\circ}\right)$ orientation of the electric field vector of the photolysis laser with the spectrometer axis. Clearly, no observable difference is found in the

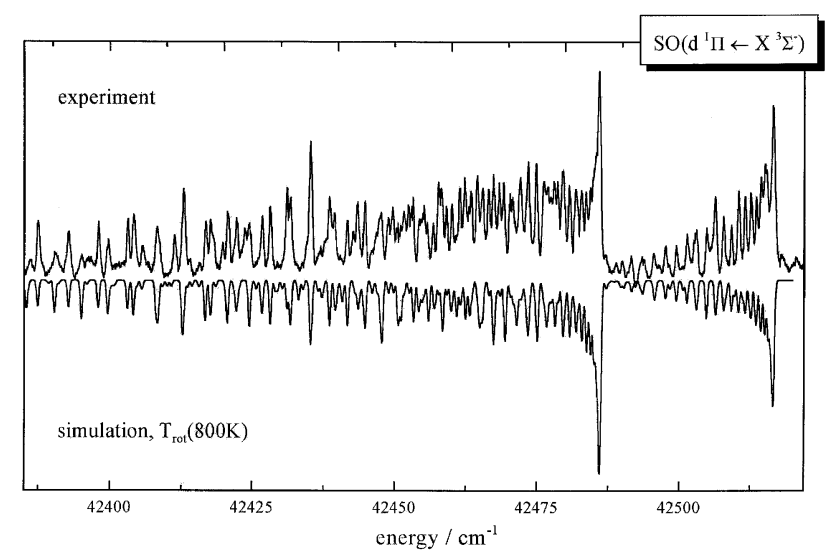

Fig. 2 Rotationally resolved spectrum of the SO fragment obtained for the one-colour dissociation between $235.2 \mathrm{~nm}$ and $235.8 \mathrm{~nm}$. Upper trace: Experimental spectrum. Lower trace: Simulation based on the $\mathrm{d}^{1} \Pi\left(v^{\prime}=0\right) \leftarrow \mathrm{X}^{3} \Sigma^{-}\left(v^{\prime \prime}=1\right)$ intercombination transition and a Boltzmann distribution at $T=800 \mathrm{~K}$. ${ }^{\mathrm{S}} \mathrm{R}_{1}$ and the $\mathrm{R}_{2}$ branches form the two bandheads at $42517 \mathrm{~cm}^{-1}(235.201 \mathrm{~nm})$ and at $42486 \mathrm{~cm}^{-1}$ $(235.371 \mathrm{~nm})$, the remaining branches do not form bandheads. Q branches are significantly less intense than $\mathrm{P}$ and $\mathrm{R}$ branches and are not distinguishable. For transitions originating from high rotational states the intensities are underestimated indicating a non-Boltzmannrotational distribution.

Table 1 Molecular constants used for the simulation of the SO $\mathrm{d}^{1} \Pi\left(v^{\prime}=0\right) \leftarrow \mathrm{X}^{3} \Sigma^{-}\left(v^{\prime \prime}=1\right)$ intercombination transition

\begin{tabular}{lcc}
\hline Molecular constants & $\mathrm{X}^{3} \Sigma^{-}\left(v^{\prime \prime}=1\right)$ & $\mathrm{d}{ }^{1} \Pi\left(v^{\prime}=0\right)$ \\
\hline$T / \mathrm{cm}^{-1}$ & 1711.700 & 44194.686 \\
$B_{\mathrm{v}} / \mathrm{cm}^{-1}$ & 0.71221 & 0.65833 \\
$D_{\mathrm{v}} / \mathrm{cm}^{-1}$ & $1.1279 \times 10^{-6}$ & $3.4 \times 10^{-6}$ \\
$\lambda / \mathrm{cm}^{-1}$ & 5.3099 & \\
$\gamma / \mathrm{cm}^{-1}$ & -0.0056577 & \\
\hline
\end{tabular}

TOF profiles for the two different polarisation directions, therefore the spatial distribution of the SO fragments must be isotropic. Moreover, the narrowness of the TOF profiles indicates slow SO fragments: Half widths at base range from $117 \mathrm{~ns}$ to $124 \mathrm{~ns}$ at a total time of flight of $12.7 \mu \mathrm{s}$, translating into maximum speeds from $1100 \mathrm{~m} \mathrm{~s}^{-1}$ to $1170 \mathrm{~m} \mathrm{~s}^{-1}$. Thus,

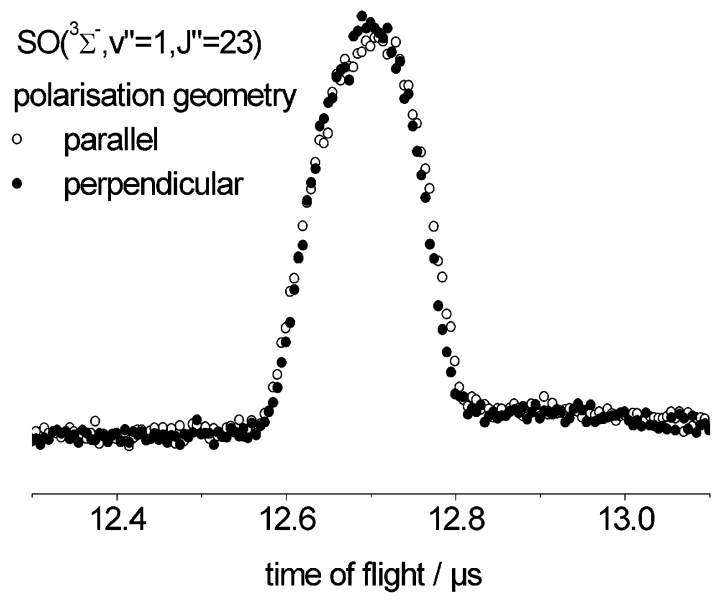

Fig. 3 Typical TOF profile of SO for an excitation wavelength of $235.756 \mathrm{~nm}\left[\mathrm{P}_{2}(23)\right]$ for both perpendicular $\left(90^{\circ}\right)$ and parallel $\left(0^{\circ}\right)$ detection geometries. Within experimental accuracy, no observable difference is found in the TOF profiles for the two geometries, indicating an isotropic spatial distribution of SO. The narrowness of the profile indicates slow SO fragments. 
maximum speeds for all observed profiles agree within experimental uncertainty. Moreover, all TOF profiles observed for different quantum states of SO exhibit an identical polarisation behaviour as the TOF profile shown in Fig. 3. Thus, we conclude at this point, without further quantitative analysis of the experimental data, that the $\operatorname{SO}(v=1)$ molecule generated in the $\mathrm{SOCl}_{2}$ fragmentation at $235.5 \mathrm{~nm}$ is released isotropically and carries only a small amount of kinetic energy.

\section{Chlorine atoms}

Chlorine atoms can be produced from $\mathrm{SOCl}_{2}$ photodissociation either via a three-body decay (1) or via the radical channel (2). For the photon energy $h \nu=42500 \mathrm{~cm}^{-1}$ the corresponding available energies are $4550 \pm 100 \mathrm{~cm}^{-1}$ and $22450 \pm 100$ $\mathrm{cm}^{-1}$, for the three-body decay (1) and the radical channel (2), respectively. For $\mathrm{Cl}^{*}$ the available energies have to be reduced by the spin-orbit energy $E_{\mathrm{S}}=882 \mathrm{~cm}^{-1}$. In analogy to the arguments presented for the $\mathrm{SO}$ fragment the $\mathrm{Cl}$ producing channels (1) and (2) can be discriminated from each other in terms of energy partitioning onto the $\mathrm{Cl}$ fragment. The $(2+1)$-REMPI detection of chlorine atoms in both spin-orbit ground $\left({ }^{2} \mathrm{P}_{3 / 2}\right)$ and excited states $\left({ }^{2} \mathrm{P}_{1 / 2}\right)$ was performed by utilizing the $4 p^{2} \mathrm{D}_{3 / 2}{ }^{0} \leftarrow 3 \mathrm{p}^{2} \mathrm{P}_{3 / 2}$ transition at $235.336 \mathrm{~nm}$ and the $4 \mathrm{p}^{2} \mathrm{~S}_{1 / 2}{ }^{0} \leftarrow 3 \mathrm{p}^{2} \mathrm{P}_{1 / 2}$ transition at $235.205 \mathrm{~nm}$. Fig. 4 shows the wavelength range from $235.17 \mathrm{~nm}$ to $235.37 \mathrm{~nm}$ containing both transitions observed in a single scan under identical conditions. Since the detection efficiencies for both spin-orbit states have been found to differ by only $6 \%,{ }^{39}$ it is clear from Fig. 4 that the production of ground state $\mathrm{Cl}$ is preferred. Although such behaviour would be expected for a purely statistical spin-orbit state distribution, the corresponding TOF profiles shown in Fig. 5 contradict the view of a simple statistical decay in that the TOF profiles exhibit pronounced differences for the two spin-orbit states both with respect to the polarisation behaviour and the profile widths. In Fig. 5 four TOF profiles are shown for both spin-orbit states and for both perpendicular and parallel polarisation geometries. All of them are richly structured. Although the presented profiles are comparable in width to the SO profiles, the significantly shorter total time of flight is proof of a pronouncedly larger kinetic energy content of the chlorine fragments in comparison with the SO fragments. For chlorine fragments an upper limit for the total time of flight is given by the presence of the two isotopes ${ }^{35} \mathrm{Cl}$ and ${ }^{37} \mathrm{Cl}$. Depending on the profile widths the acceleration voltage must be adjusted such that the two isotopically pure profiles do not overlap. Ground state chlorine exhibits a narrow central peak for both polarisation geometries which is

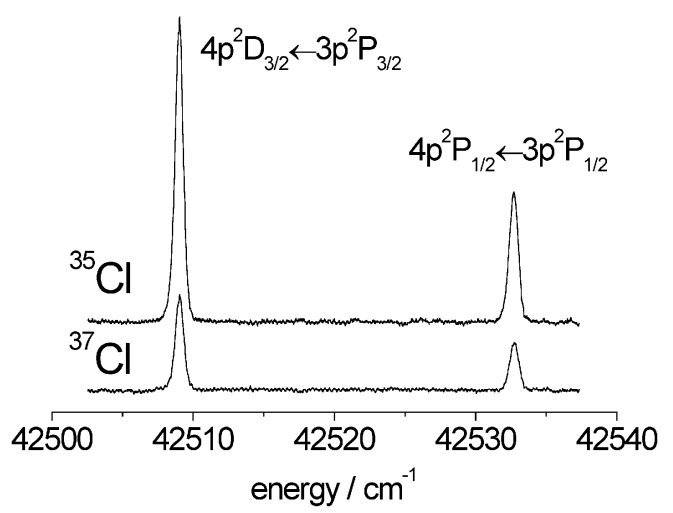

Fig. 4 The $(2+1)$-REMPI signals for ground state atoms $(4 \mathrm{p}$ ${ }^{2} \mathrm{D}_{3 / 2}{ }^{0} \leftarrow 3 \mathrm{p}^{2} \mathrm{P}_{3 / 2}$ transition at $235.336 \mathrm{~nm}$ ) and excited spin-orbit state atoms $\left(4 \mathrm{p}{ }^{2} \mathrm{~S}_{1 / 2} \leftarrow 3 \mathrm{p}^{2} \mathrm{P}_{1 / 2}\right.$ transition at $\left.235.205 \mathrm{~nm}\right)$ indicate a preferred production of ground state $\mathrm{Cl}$ because the transition probabilities differ by only $6 \%$ and the laser energy is constant over the tuning range.
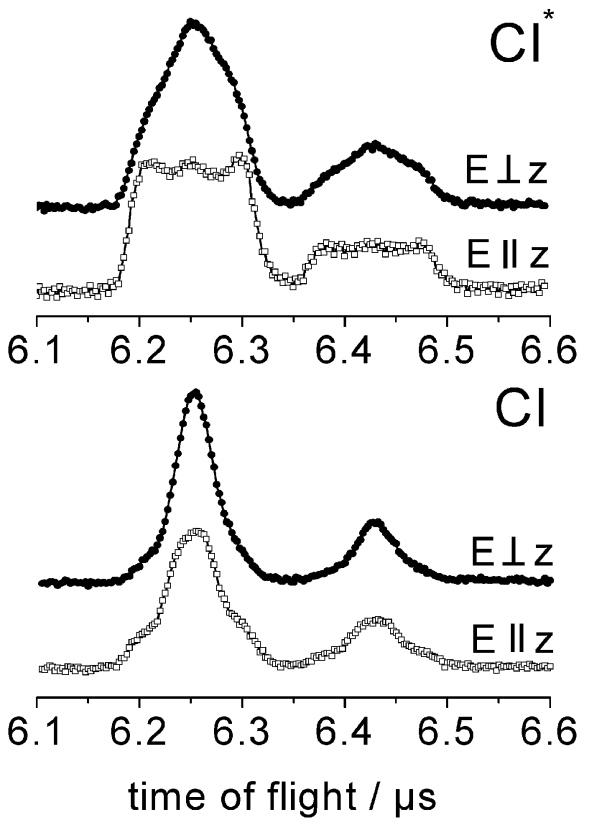

Fig. 5 Chlorine atom TOF profiles for both spin-orbit states and for both perpendicular and parallel polarisation geometries. Ions appearing in the wing of the profiles possess a large velocity component $v_{z}$ along the spectrometer axis while ions appearing at the centre of the profile either move perpendicularly to the spectrometer axis, or they are simply slow. The complex behaviour results from a bimodal speed distribution with two different positive anisotropy parameters. The smaller TOF profiles at slightly larger times-of-flight are the signature of the ${ }^{37} \mathrm{Cl}$ isotope.

accompanied by small shoulders on both sides. The central peak is larger and the shoulders are less pronounced for the perpendicular geometry than for the parallel geometry. In this context it is important to note that ions appearing in the wing of the profiles possess a large velocity component $v_{z}$ along the spectrometer axis while ions appearing at the centre of the profile either move perpendicularly to the spectrometer axis, or they are simply slow. In principle, the TOF profiles for the excited spin-orbit state seem to be composed of the same features, but with comparable contributions instead of a dominating central peak. Consequently, upon changing the polarisation geometry the central peak, which dominates the perpendicular case, plays a minor role only in the parallel case. The smaller TOF profiles at slightly larger times-of-flight are the signature of the ${ }^{37} \mathrm{Cl}$ isotope and exhibit identical behaviour to the profiles of the ${ }^{35} \mathrm{Cl}$ isotope with its threefold larger natural abundance. Qualitatively, the complexity of the TOF profiles shown in Fig. 5 is caused by three main effects. First, on average, ground state $\mathrm{Cl}$ is released with significantly less kinetic energy than spin-orbit state excited $\mathrm{Cl}^{*}$ although the maximum kinetic energies are similar. Second, as an experimental fact, atoms in both spin-orbit states move preferentially along the polarisation vector of the photolysis laser. Thus, for the parallel case the TOF profiles appear broader than for the perpendicular case. Third, the profiles reflect some sort of bimodality in the $\mathrm{Cl}$ atom speed distributions which must be specific to the $\mathrm{Cl}$ quantum state. Very similar effects have been observed in the UV photodissociation of phosgene. $^{4,15,16}$

\section{Discussion}

\section{The SO spectrum}

The lower trace in Fig. 2 shows a simulation of the $\mathrm{d}^{1} \Pi$ $\left(v^{\prime}=0\right) \leftarrow \mathrm{X}^{3} \Sigma^{-}\left(v^{\prime \prime}=1\right)$ intercombination transition sug- 
gested by Archer et al. ${ }^{31}$ using the constants given in Table 1 and a temperature parameter of $800 \mathrm{~K}$. The agreement between the simulation and the experiment is good. The ${ }^{\mathrm{S}} \mathrm{R}_{1}$ and the $\mathrm{R}_{2}$ branches form the two bandheads at $42517 \mathrm{~cm}^{-1}$ $(235.201 \mathrm{~nm})$ and at $42486 \mathrm{~cm}^{-1}(235.371 \mathrm{~nm})$, the remaining branches do not form bandheads. The three $\mathrm{Q}$ branches are significantly less intense than the $\mathrm{P}$ and $\mathrm{R}$ branches and are therefore not distinguishable in the experimental spectrum. For transitions originating from high rotational states the intensities are underestimated, indicating a non-Boltzmann rotational distribution. For comparison a similar simulation was carried out assuming the transition to be $\mathrm{B}^{\prime}$ ${ }^{3} \Sigma^{-}\left(v^{\prime}=0\right) \leftarrow \mathrm{X}^{3} \Sigma^{-}\left(v^{\prime \prime}=1\right)$ as was proposed by Braatz and Tiemann. ${ }^{37}$ Constants were used as reported by the authors. The agreement between simulation and experiment is similar to the previous case if a temperature parameter of $700 \mathrm{~K}$ is used in the simulation. The difference in the temperature parameters results from different Hönl-London factors for the two transitions. ${ }^{40}$ Proposing the latter transition was mainly motivated by two reasons: because intercombination $(\Delta S \neq 0)$ transitions are spin-forbidden and because of the apparent absence of Q branches. However, spin-orbit coupling may be responsible for a non-vanishing probability for the intercombination transition and Hönl-London factors for the Q branches of the intercombination transition are almost one order of magnitude smaller than their counterparts for the $\mathrm{P}$ and $\mathrm{R}$ branches. ${ }^{40}$ Therefore, the appearance of the intercombination transition is very similar to the appearance of a $\Sigma-\Sigma$ transition. An unequivocal assignment of the transition cannot be obtained from our experimentally observed spectrum. However, based on the arguments presented above and since the d ${ }^{1} \Pi\left(v^{\prime}=0\right) \leftarrow \mathrm{X}^{3} \Sigma^{-}\left(v^{\prime \prime}=1\right)$ transition is predicted on the basis of ab initio calculations, ${ }^{31}$ the intercombination transition most likely is the origin of the observed spectrum.

\section{SO speed and spatial distribution}

TOF profiles such as the one shown in Fig. 3 were monitored for $J=11,18,22$, and 23 employing the transitions listed in Table 2. Whereas the half width at base $\Delta t$ of the TOF profiles $P(t)$ is determined by the maximum speed $v_{\max }$ of the fragments obtained in the dissociation process according to eqn. (4), the shape of $P(t)$ reflects the spatial fragment distribution $P(v, \theta){ }^{41}$ which can be characterised by the anisotropy parameter $\beta$ :

$$
P(v, \theta) \propto 1+\beta(v) P_{2}(\cos \theta)
$$

Here, $\theta$ is the angle of the fragment recoil velocity $v$ with the polarisation vector of the dissociating laser and $P_{2}$ is the second Legendre polynomial. $\beta$ ranges from -1 to +2 , describing an isotropic distribution for $\beta=0$, a $\cos ^{2} \theta$ distribution for

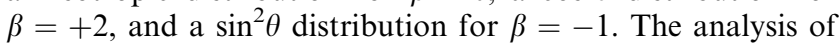
the profiles yields maximum SO speeds of $1150 \pm 75 \mathrm{~m} \mathrm{~s}^{-1}$ and $\beta_{\text {SO }}$ parameters of $0.0 \pm 0.2$ for all five TOF profiles regardless of the probed quantum state.

Generally, energy partitioning in a three-body decay of an ABC parent molecule is as difficult to analyse as to model. ${ }^{3,4}$ Fortunately, in order to calculate maximum kinetic energies

Table 2 Selected transitions used to monitor TOF profiles. Transitions marked by an asterisk occur at the bandheads

\begin{tabular}{ll}
\hline Probed transition & Transition wavelength $\lambda / \mathrm{nm}$ \\
\hline $\mathrm{R}_{2}(11)^{*}$ & 235.371 \\
$\mathrm{O}_{3}(18)$ & 235.751 \\
$\mathrm{P}_{2}(22)$ & 235.734 \\
$\mathrm{P}_{2}(23)$ & 235.756 \\
${ }^{\mathrm{S}} \mathrm{R}_{1}(23)^{*}$ & 235.201 \\
\hline
\end{tabular}

that can be transferred onto fragment $\mathrm{A}$ it is sufficient to look at limiting cases. One condition for maximum kinetic energy transfer is that all three fragments $\mathrm{A}, \mathrm{B}$, and $\mathrm{C}$ move along a common axis, so that momentum conservation does not require kinetic energy to be lost in the remaining space dimensions. Second, also based on momentum considerations, for large energy transfer, fragment $\mathrm{A}$ will have to move in the opposite direction to the two partner fragments $\mathrm{B}$ and $\mathrm{C}$. Third, fragment A's kinetic energy is maximised (i.e. the combined kinetic energies of the two partner fragments are minimised) if $\mathrm{B}$ and $\mathrm{C}$ move at exactly the same speed. Then, the situation is identical to a two-body decay where the two partners form an unbound "quasi-molecule", and the maximum kinetic energy becomes $E_{\text {kin }}{ }^{\max }(\mathrm{A})=\frac{m_{\mathrm{BC}}}{M} E_{\mathrm{av}}$, where $M$ is the mass of the parent molecule $\mathrm{ABC}$ and $m_{\mathrm{BC}}$ is the combined mass of the partner fragments $\mathrm{B}$ and $\mathrm{C}$. For a synchronous concerted three-body decay this situation corresponds to a quasi-linear configuration with a decay angle of $0^{\circ}$ or $360^{\circ}$, for a purely sequential decay through a long-lived intermediate $\mathrm{AB}$ this situation corresponds to a forward scattering of $\mathrm{A}$ at a unique energy deposition $E_{\mathrm{AB}} *$ in the intermediate $\mathrm{AB}$ for which the relationship $\mathrm{d} E_{\mathrm{kin}}(\mathrm{A}) / \mathrm{d} E_{\mathrm{AB}}=0$ holds. Applying this condition to eqn. (6) in ref. (4) yields the intuitively derived result given above.

The available energy for the three-body dissociation into ground state products (1), was calculated to be $4550 \pm$ $150 \mathrm{~cm}^{-1} .^{22}$ Then, the maximum kinetic energy that can be obtained by an SO fragment in reaction (1) is $E_{\mathrm{kin}}{ }^{\max }(\mathrm{SO})=\frac{m_{\mathrm{Cl}, \mathrm{Cl}}}{M} E_{\mathrm{av}}=\frac{70}{1,18} E_{\mathrm{av}}$, i.e. $2700 \pm 90 \mathrm{~cm}^{-1}$ for $v^{\prime \prime}=0$ and $2020 \pm 90 \mathrm{~cm}^{-1}$ for $v^{\prime \prime}=1$, corresponding to maximum speeds of $1150 \pm 30 \mathrm{~m} \mathrm{~s}^{-1}$ and $1000 \pm 30 \mathrm{~m} \mathrm{~s}^{-1}$, respectively. ${ }^{3,4}$ Here, formation of two ground state ${ }^{35} \mathrm{Cl}$ fragments and the vibrational quantum $\omega_{\mathrm{SO}(\mathrm{X})}=1150.7 \mathrm{~cm}^{-1}$ were considered. ${ }^{42}$ For the molecular channel (3), the available energy is increased by the energy of the $\mathrm{Cl}-\mathrm{Cl}$ bond in the $\mathrm{Cl}_{2}$ molecule and therefore amounts to $24550 \pm 150 \mathrm{~cm}^{-1}$. If $\mathrm{Cl}_{2}$ is generated in the $\mathrm{X}^{1} \Sigma_{\mathrm{g}}+$ ground state, the corresponding maximum SO kinetic energy is $14560 \pm 90 \mathrm{~cm}^{-1}$, and the maximum speed is $2700 \pm 20 \mathrm{~m} \mathrm{~s}^{-1}$. For $\mathrm{Cl}_{2}$ formation in the $\mathrm{B}^{3} \Pi_{0}{ }^{+}$u state at $\nu_{00}=17658 \mathrm{~cm}^{-1}$ the available energy amounts to $6890 \pm 150$ $\mathrm{cm}^{-1}$, the maximum kinetic energy becomes $4090 \pm 90 \mathrm{~cm}^{-1}$ and the maximum speed equals $1430 \pm 30 \mathrm{~m} \mathrm{~s}^{-1}$. Secondary photolysis of a primary SOCl radical fragment upon absorption of a second photon would result in an even higher maximum speed for the final SO fragment than for the molecular channel with $\mathrm{Cl}_{2}\left(\mathrm{X}^{1} \Sigma_{\mathrm{g}}{ }^{+}\right)$as partner. Uncertainties include contributions from uncertainties in the dissociation enthalpies and thermal parent molecule motion due to incomplete cooling in the supersonic jet expansion $(10 \mathrm{~K})$. Clearly, the experimentally determined value of $1150 \pm 75 \mathrm{~m} \mathrm{~s}^{-1}$ agrees best with the three-body decay channel (1) whereas the molecular channel (3) with a $\mathrm{Cl}_{2}\left(\mathrm{X}^{1} \Sigma_{\mathrm{g}}{ }^{+}\right)$partner as well as a secondary photolysis of $\mathrm{SOCl}$ can be ruled out, even after allowing for significant internal excitation of $\mathrm{Cl}_{2}$. The possibility of producing electronically excited $\mathrm{Cl}_{2}\left(\mathrm{~B}^{3} \Pi_{0}{ }_{\mathrm{u}}\right)$ together with ground state $\mathrm{SO}(\mathrm{X}$ ${ }^{3} \Sigma^{-}$) has been discussed before for a dissociation wavelength of $248 \mathrm{~nm}$. However, at this wavelength the main contribution to the minor $(c a .5 \%)$ molecular channel consists of $\mathrm{Cl}_{2}(\mathrm{X}$ $\left.{ }^{1} \Sigma_{\mathrm{g}}^{+}\right)$in conjunction with $\mathrm{SO}\left(\mathrm{b}^{1} \Sigma^{+}\right)$, a channel which is not being monitored in our experimental set-up. A contribution of the $\mathrm{Cl}_{2}(\mathrm{~B})+\mathrm{SO}(\mathrm{X})$ molecular channel is not unlikely in our case for those SO fragments produced with speeds larger than $1000 \mathrm{~m} \mathrm{~s}^{-1}$. The majority of the observed SO fragments, however, is slower than this value and must be associated with a three-body decay, since $\mathrm{Cl}_{2}(\mathrm{~B})$ correlates to $\mathrm{Cl}+\mathrm{Cl}^{*}$ and has no internal degrees of freedom where internal energy above the dissociation limit could be stored. On the other hand, the vanishing $\beta$ parameter indicates a long-lived species that survives one or several rotational periods prior to its final decay. Therefore, most likely this species is a highly excited $\mathrm{SOCl}^{*}$ fragment 
that unimolecularly decays into the final $\mathrm{SO}$ and $\mathrm{Cl}$ products. In view of the small available energy it is not surprising that the time until this triatomic system arrives at the exit channel to the final $\mathrm{SO}$ and $\mathrm{Cl}$ fragments is long enough for the $\mathrm{SOCl}^{*}$ intermediate to forget the initial alignment of the $\mathrm{SOCl}_{2}$ parent molecule induced by the absorption of the photon. These results also agree with the conclusions drawn from the 193 $\mathrm{nm}$ photolysis of $\mathrm{SOCl}_{2}$, where the three-body channel was found to dominate the dissociation process, while at $248 \mathrm{~nm}$ the radical channel (2) was most important, accompanied by the molecular channel (3) to a small extent. ${ }^{22-24}$ Other than at $235 \mathrm{~nm}$, where the three-body decay is most probably sequential, at $193 \mathrm{~nm}$ it proceeds in an asynchronous concerted fashion, characterised by a negative $\beta_{\text {So }}$ parameter and a positive $\beta_{\mathrm{Cl}}$ parameter. ${ }^{22,23}$ This difference is easily rationalised by the significantly higher available energy, allowing the system to access the $\mathrm{SOCl}^{*}$ exit channel in a much shorter time than in the $235 \mathrm{~nm}$ case, such that the mean lifetime of the $\mathrm{SOCl}^{*}$ intermediate becomes shorter than its mean rotational period. Based on the observed anisotropies at $193 \mathrm{~nm}$ a fast decay on the excited potential energy surface was suggested. Whether the reduced $\beta_{\text {SO }}$ parameter at $235 \mathrm{~nm}$ is due to a long lifetime of the $\mathrm{SOCl}^{*}$ intermediate produced at $235 \mathrm{~nm}$ or whether an excited potential energy surface of another symmetry is involved in the $235 \mathrm{~nm}$ dissociation, cannot be decided on the basis of the vanishing $\beta_{\text {So }}$ parameter. A more detailed picture of the decay process requires the analysis of the properties of the nascent $\mathrm{Cl}$ atoms from the $\mathrm{SOCl}_{2}$ dissociation.

\section{Energy partitioning}

Chlorine atoms in the ${ }^{2} \mathrm{P}_{3 / 2}$ ground state $(\mathrm{Cl})$ or in the ${ }^{2} \mathrm{P}_{1 / 2}$ excited spin-orbit state $\left(\mathrm{Cl}^{*}\right)$ can be produced either by the three-body channel (1), which has been found to be operative at $235 \mathrm{~nm}$ in the preceding section and to be the dominant channel at $193 \mathrm{~nm},{ }^{2-24}$ or by the radical channel (2), which is almost exclusively responsible for the $248 \mathrm{~nm}$ dissociation of $\mathrm{SOCl}_{2} \cdot{ }^{22-24}$

The state-specific TOF profiles shown in Fig. 5 were converted into the two kinetic energy distributions shown in Fig. 6 by a least squares fit routine ${ }^{25}$ taking into account the spatial fragment anisotropy which manifests itself in the polarisation dependent shapes of the profiles. The procedure is outlined in more detail in the Appendix. Both spin-orbit state specific kinetic energy distributions are bimodal, but differ significantly in the individual contributions of slow and fast $\mathrm{Cl}$ fragments to the overall process. Based on the available energies for the three-body decay (1) and the radical channel (2), maximum $\mathrm{Cl}$ fragment kinetic energies of $3190 \pm 70 \mathrm{~cm}^{-1}$ and $15790 \pm 70 \mathrm{~cm}^{-1}\left(2570 \pm 70 \mathrm{~cm}^{-1}\right.$ and $\left.15170 \pm 70 \mathrm{~cm}^{-1}\right)$ are obtained, corresponding to maximum speeds of $1480 \pm 20 \mathrm{~m} \mathrm{~s}^{-1}$ and $3290 \pm 10 \mathrm{~m} \mathrm{~s}^{-1}\left(1330 \pm 20 \mathrm{~m} \mathrm{~s}^{-1}\right.$ and $3225 \pm 10 \mathrm{~m} \mathrm{~s}^{-1}$ ) where the bracketed values are for $\mathrm{Cl}^{*}$. The three-body regions extending from $0 \mathrm{~cm}^{-1}$ to $3190 \mathrm{~cm}^{-1}$ $\left(2570 \mathrm{~cm}^{-1}\right)$ are shaded in Fig. 6. For ground state $\mathrm{Cl}$, the two components which are responsible for the observed bimodality in the kinetic energy distributions can very well be associated with the three- and two-body decay channels. Although less clearly separated, the same two components, but with different intensity, comprise the kinetic energy distribution for spin-orbit excited $\mathrm{Cl}^{*}$.

For the two-body region above $3190 \mathrm{~cm}^{-1}\left(2570 \mathrm{~cm}^{-1}\right)$, where the chlorine fragment is associated with a stable $\mathrm{SOCl}$ radical the internal energy distribution of the $\mathrm{SOCl}$ fragment can be extracted from the kinetic energy distributions of Fig. 6, as shown in Fig. 7. Basically, Fig. 7 inverts Fig. 6 according to $E_{\text {int }}(\mathrm{SOCl})=E_{\mathrm{av}}-E_{\text {kin }}(\mathrm{Cl})-E_{\text {kin }}(\mathrm{SOCl})$ where the kinetic energies $E_{\mathrm{kin}}(\mathrm{SOCl})$ and $E_{\mathrm{kin}}(\mathrm{Cl})$ are related to each other by the $\mathrm{Cl} / \mathrm{SOCl}$ mass ratio due to the conservation of linear momentum. Only $E_{\text {kin }}(\mathrm{Cl})$ values above $3190 \mathrm{~cm}^{-1}$

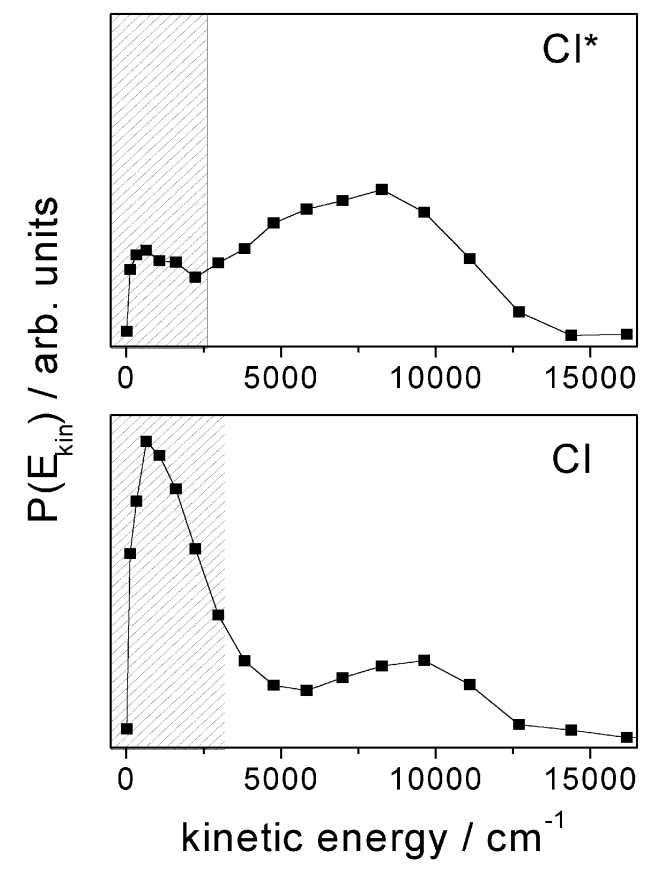

Fig. 6 Chlorine atom kinetic energy distributions derived from the TOF profiles of Fig. 5. For $\mathrm{Cl}$ the three-body region extends from 0 $\mathrm{cm}^{-1}$ to $3190 \mathrm{~cm}^{-1}$ and the two-body region from $3190 \mathrm{~cm}^{-1}$ to $15790 \mathrm{~cm}^{-1}$. The two components which are responsible for the bimodality can very well be associated with the three- and two-body decay channels. The three-body region is marked by a shaded area. For $\mathrm{Cl}^{*}$, the three-body region extends from $0 \mathrm{~cm}^{-1}$ to $2570 \mathrm{~cm}^{-1}$ and the two-body region from $2570 \mathrm{~cm}^{-1}$ to $15170 \mathrm{~cm}^{-1}$.

$\left(2570 \mathrm{~cm}^{-1}\right)$ have been taken into account. The internal energy is broadly distributed from 0 to the dissociation limit $\left(D_{0}(\mathrm{SOCl})=17900 \pm 150 \mathrm{~cm}^{-}\right)^{22}$ where the three-body region starts. The mean internal energy is $c a$. $50 \%$ of the available

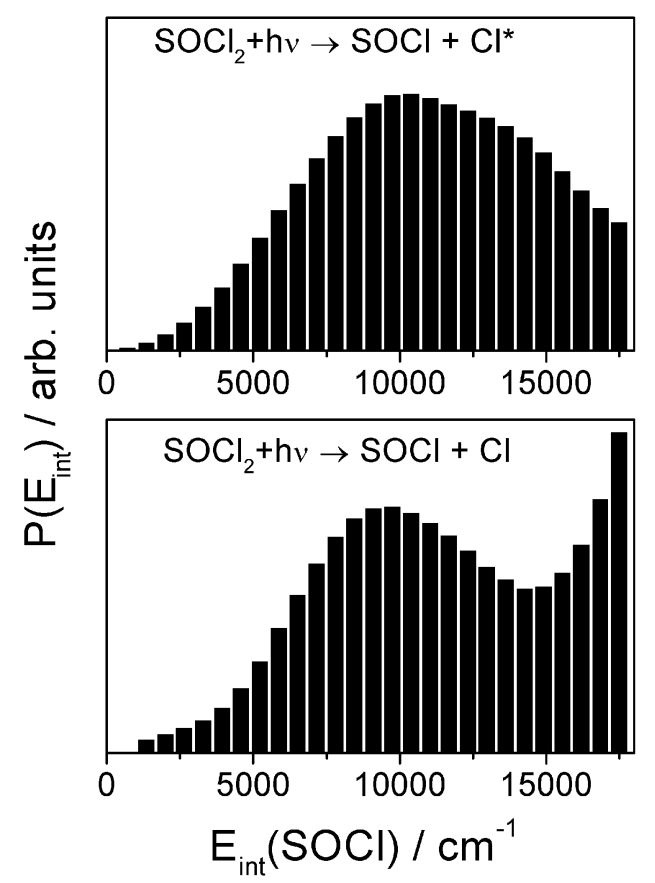

Fig. 7 Internal energy distribution of the stable SOCl fragment extracted from the kinetic energy distributions of Fig. 6. The mean internal energy is $c a .50 \%$ of the available energy. The internal energy is broadly distributed from 0 to the dissociation limit where the threebody region begins, most probably as vibrational and rotational excitation. 
energy. The exact nature of this internal excitation remains unclear. It has been suggested that a low-lying electronic state might be responsible for this excitation. ${ }^{22}$ However, ab initio calculations yielded no evidence of such a low-lying electronic state. ${ }^{43}$ Therefore, the most probable explanation is significant vibrational and rotational excitation of the $\mathrm{SOCl}$ radical. This behaviour is not uncommon and has also been found in the photodissociation of $\mathrm{ClO}_{2} \cdot{ }^{44}$ The large values of the internal energy distribution at $16000 \mathrm{~cm}^{-1}$ to $18000 \mathrm{~cm}^{-1}$ for ground state $\mathrm{Cl}$ are most likely a consequence of the contribution of the electronic state associated with three-body decay into SO and two ground state $\mathrm{Cl}$ atoms, as discussed in the preceding section. This scenario might be called an inhibited three-body decay: If the energy in the $\mathrm{SOCl}^{*}$ intermediate is just not high enough to dissociate it, it will manifest itself in exactly the way seen for the high internal energy region of $\mathrm{SOCl}$ associated with the production of a ground state chlorine atom. This feature is absent in the internal energy distribution of $\mathrm{SOCl}$ associated with the spin-orbit excited state because the three-body decay was found to predominantly generate ground state $\mathrm{Cl}$ atoms.

\section{Fragment anisotropies}

The assignment made for the kinetic energy distributions in the preceding section is supported by the behaviour of the anisotropy parameter $\beta_{\mathrm{Cl}}$ which rises sharply from a small positive value of $0.2 \pm 0.2$ in the three-body region to a significantly larger value of $0.8 \pm 0.2$ in the two-body region. An overview of the properties and abundances of the components is given in Table 3. The observed anisotropy behaviour in the high kinetic energy regime (two-body decay) agrees excellently with the previously observed anisotropy of the radical channel (2) at $248 \mathrm{~nm}$, while the low kinetic energy results (three-body decay) coincide with the $193 \mathrm{~nm}$ three-body decay results. ${ }^{22,23}$

Thus, for the dissociation at $235 \mathrm{~nm}$, a simultaneous excitation of two potential energy surfaces (PES) is very likely, as expected from the excitation of $\mathrm{SOCl}_{2}$ in a spectral region characterised by overlapping absorption bands. The lower surface mainly produces $\mathrm{SOCl}+\mathrm{Cl}$ and the higher one mainly generates $\mathrm{SO}+2 \mathrm{Cl}$. The different $\beta_{\mathrm{Cl}}$ parameters hint at different symmetries of the two involved PES. The contributions of the channels are 59\% for the two-body (radical) decay and $41 \%$ for the three-body decay.

The observed $\beta_{\mathrm{Cl}}$ parameter of 0.8 for the two-body radical decay (2) can only be obtained if the upper PES is of $A^{\prime \prime}$ symmetry. The symmetry plane contains the $\mathrm{S}-\mathrm{O}$ double bond and bisects the $\mathrm{Cl}-\mathrm{S}-\mathrm{Cl}$ bond angle of $97^{\circ}$ in the $\mathrm{X}{ }^{1} \mathrm{~A}^{\prime}$ ground state $^{45}$ Therefore, a dipole moment of a" symmetry connecting the $\mathrm{Cl}$ atoms forms a recoil angle $\alpha$ of $41.5^{\circ}$ with the recoil direction of the $\mathrm{Cl}$ atoms if the fragmentation proceeds instantaneously from the ground state geometry. According to

$$
\beta_{\mathrm{Cl}}=2 P_{2}(\cos \alpha)
$$

the expected $\beta_{\mathrm{Cl}}$ parameter for this recoil angle is 0.7 . An increased $\beta_{\mathrm{Cl}}$ parameter can only be observed if the excited state geometry differs from the ground state geometry for which the calculation was performed in that an increased bond

Table 3 Anisotropy parameters and abundances of chlorine atoms released via different dissociation channels. $\left.\mathrm{Cl}: \mathrm{Cl}^{2} \mathrm{P}_{3 / 2}\right), \mathrm{Cl}^{*}$ : $\mathrm{Cl}\left({ }^{2} \mathrm{P}_{1 / 2}\right)$, R1: $\mathrm{SOCl}_{2}+\mathrm{h} \nu \rightarrow \mathrm{SO}+2 \mathrm{Cl}, \mathrm{R} 2: \mathrm{SOCl}_{2}+\mathrm{h} \nu \rightarrow \mathrm{SOCl}+\mathrm{Cl}$

\begin{tabular}{lll}
\hline & $\beta$ & Abundance(\%) \\
\hline $\mathrm{Cl}(\mathrm{R} 2)$ & $0.8 \pm 0.2$ & 30 \\
$\mathrm{Cl}(\mathrm{R} 1)$ & $0.2 \pm 0.2$ & 37 \\
$\mathrm{Cl}^{*}(\mathrm{R} 2)$ & $0.8 \pm 0.2$ & 29 \\
$\mathrm{Cl}^{*}$ (R1) & $0.2 \pm 0.2$ & 4 \\
\hline
\end{tabular}

angle leads to a decreased recoil angle. However, the observed $\beta_{\mathrm{Cl}}$ value of $0.8 \pm 0.2$ agrees within the experimental uncertainty with the value calculated for an instantaneous decay from a molecular geometry which is similar to the ground state geometry.

Excitation to a state of $\mathrm{A}^{\prime}$ symmetry may result in $\beta$ parameters ranging from -1.0 to +0.3 if only symmetry is considered, again based on an instantaneous decay from ground state geometry. The experimentally obtained three-body decay anisotropy parameter $\beta_{\mathrm{Cl}}=0.2 \pm 0.2$ lies inside the range that can be associated with an excitation to an $\mathrm{A}^{\prime}$ surface. This assignment agrees well with the $\beta_{\text {SO }}$ parameter of -0.65 observed in the dissociation at $193 \mathrm{~nm}$, although from the following considerations a simultaneous excitation to several excited states is not unlikely.

In the molecular orbital picture, the $\mathrm{SOCl}_{2}$ molecule has 26 valence electrons: eight bonding electrons forming the $\sigma_{\mathrm{SO}}$, the $\pi_{\mathrm{SO}}$ and the two $\sigma_{\mathrm{SCl}}$ bonds, two lone pairs at the $\mathrm{O}$ atom, one lone pair at the $\mathrm{S}$ atom, and six lone pairs at the $\mathrm{Cl}$ atoms.

$$
\begin{aligned}
\underbrace{\left(7 \mathrm{a}^{\prime \prime}\right)^{2}\left(11 \mathrm{a}^{\prime}\right)^{2}\left(12 \mathrm{a}^{\prime}\right)^{2}\left(13 \mathrm{a}^{\prime}\right)^{2}}_{\text {bonds }} \underbrace{\left(8 \mathrm{a}^{\prime \prime}\right)^{2}\left(14 \mathrm{a}^{\prime}\right)^{2}\left(15 \mathrm{a}^{\prime}\right)^{2}\left(9 \mathrm{a}^{\prime \prime}\right)^{2}}_{\mathrm{n}_{\mathrm{o}}, \mathrm{n}_{\mathrm{cl}}(3 \mathrm{~s})} \\
\times \underbrace{\left(16 \mathrm{a}^{\prime}\right)^{2}\left(17 \mathrm{a}^{\prime}\right)^{2}\left(10 \mathrm{a}^{\prime \prime}\right)^{2}\left(11 \mathrm{a}^{\prime \prime}\right)^{2}}_{\mathrm{n}_{\mathrm{cl}}(3 \mathrm{p})} \underbrace{\left(18 \mathrm{a}^{\prime}\right)^{2}}_{\mathrm{n}_{\mathrm{s}}}
\end{aligned}
$$

The large number of non-bonding $\mathrm{S}(3 \mathrm{p})$ and $\mathrm{Cl}(3 \mathrm{p})$ electrons gives rise to several transitions $\sigma^{*} \mathrm{SCl} \leftarrow \mathrm{n}_{\mathrm{S}} / \mathrm{n}_{\mathrm{Cl}}$ and the $\pi^{*}{ }_{\mathrm{SO}} \leftarrow \mathrm{n}_{\mathrm{S}} / \mathrm{n}_{\mathrm{Cl}}$ of both $\mathrm{A}^{\prime}$ and $\mathrm{A}^{\prime \prime}$ symmetry located in a narrow energy range and giving rise to the absorption spectrum of Fig. 1. The spectrum has been fitted by three overlapping Gaussian absorption features labelled A, B, and C, centred at $253 \mathrm{~nm}, 235 \mathrm{~nm}$, and $191 \mathrm{~nm}$, respectively. The first transition A is probably associated with the molecular decay (3) occurring on a PES of $\mathrm{A}^{\prime}$ symmetry and might be identified with the $\pi^{*}$ so $\leftarrow \mathrm{n}_{\mathrm{S}}$ transition. The second transition $\mathrm{B}$, the main one accessed in our experiment, produces mainly chlorine atoms in both spin-orbit states via the radical channel (2) in conjunction with $\mathrm{SOCl}$ radicals and is of $\mathrm{A}^{\prime \prime}$ symmetry. The third transition $\mathrm{C}$ is associated with a three-body decay (1) and exhibits $\mathrm{A}^{\prime}$ symmetry. It produces mainly (88\%) two slow ground state $\mathrm{Cl}$ atoms. Experimental evidence was found in photodissociation experiments at $193 \mathrm{~nm}$ for some contribution of another state of $\mathrm{A}^{\prime \prime}$ symmetry.

\section{Spin-orbit state specificity}

Integrating the areas under the $\mathrm{Cl}$ and the $\mathrm{Cl}^{*}$ profiles shown in Fig. 4 results in an intensity ratio $I\left(\mathrm{Cl}^{*}\right) / I(\mathrm{Cl})=0.5$. Taking into account the detection sensitivity for the $\mathrm{Cl}$ spin-orbit states an overall branching ratio of $P\left(\mathrm{Cl}^{*}\right) / P(\mathrm{Cl})=$ $0.53 \pm 0.08$ is obtained, corresponding to a $\mathrm{Cl}^{*}$ yield $\phi\left(\mathrm{Cl}^{*}\right)=P\left(\mathrm{Cl}^{*}\right) /\left[P(\mathrm{Cl})+P\left(\mathrm{Cl}^{*}\right)\right]=0.35+0.05$. This value is used to weight the kinetic energy distributions of Fig. 6 with respect to each other. The agreement of the determined branching ratio with the purely statistical value of 0.33 is pure coincidence, however, and in reality the dissociation proceeds in a highly non-statistical manner giving rise to the characteristic state-specific profile shapes of Fig. 5. From the values given in Table 3 individual branching ratios $\phi_{1}\left(\mathrm{Cl}^{*}\right)$ and $\phi_{2}\left(\mathrm{Cl}^{*}\right)$ can be calculated for the three-and the two-body mechanisms (1) and (2), each representing the excitation into one of the two different PES discussed in the preceding section: $\phi_{1}\left(\mathrm{Cl}^{*}\right)=0.10$ and $\phi_{2}\left(\mathrm{Cl}^{*}\right)=0.49$. The overall, nearly 'statistical' branching ratio of 0.35 is then obtained by averaging the individual branching ratios weighted with the contribution of the respective channel.

The quality of the fitting routine is demonstrated in Fig. 8 where the result of the least squares procedure is presented for all four TOF profiles of Fig. 5. Here, the individual contri- 

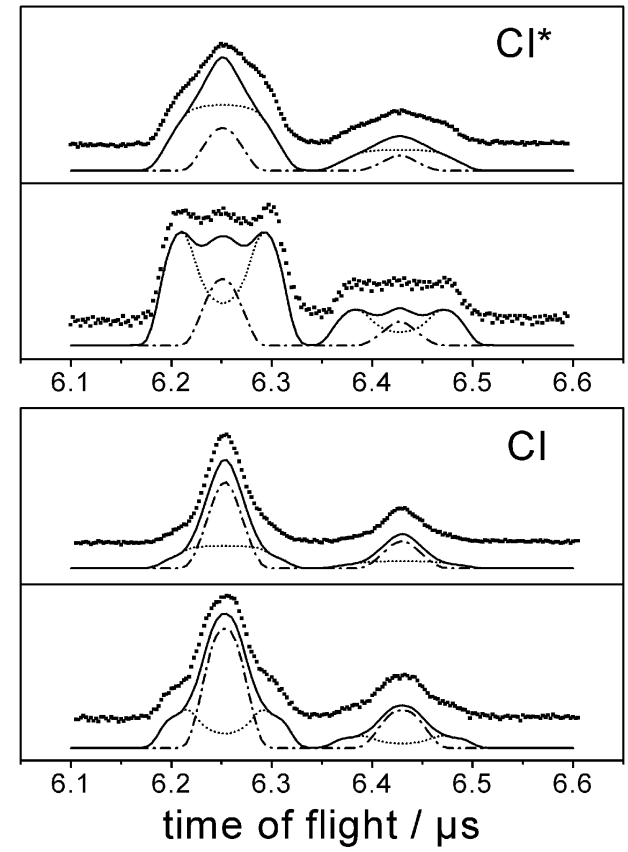

Fig. 8 Result of the least squares fit procedure for all four TOF profiles of Fig. 5. Individual contributions of two- and three-body decay channels to the spin-orbit state specific profile shapes are shown for both parallel and perpendicular polarisation geometries (symbols: experimental data, dotted line; two-body decay, dash-dotted line; three-body decay, solid line; sum of two- and three-body decay). Upper traces were obtained for a perpendicular polarisation geometry, lower ones for parallel polarisation geometry.

butions of two- and three-body decay channels to the spinorbit state specific profile shapes are shown for both parallel and perpendicular polarisation geometries.

\section{E. Conclusion}

The photodissociation of $\mathrm{SOCl}_{2}$ at a dissociation wavelength of $235 \mathrm{~nm}$ proceeds via the simultaneous excitation of two potential energy surfaces of $\mathrm{A}^{\prime \prime}$ and of $\mathrm{A}^{\prime}$ symmetry, respectively. Both correspond to a direct excitation of the dissociation coordinate and are therefore expected to lead to fast and direct dissociation. This behaviour distinguishes the $\mathrm{SOCl}_{2}$ dissociation from the dissociation of structurally similar molecules like $\mathrm{COCl}_{2}$ or $\mathrm{CSCl}_{2}$. The $\mathrm{A}^{\prime \prime}$ state produces equal amounts of ground and excited spin-orbit state chlorine atoms in conjunction with a stable $\mathrm{SOCl}$ radical which carries about $50 \%$ of the available energy as internal energy. The spatial fragment distribution can be described by an anisotropy parameter of $0.8 \pm 0.2$, implying an instantaneous decay from ground state geometry. The $\mathrm{A}^{\prime}$ state mainly produces three fragments, $\mathrm{SO}+2 \mathrm{Cl}$, via a sequential three-body decay. Here, predominantly ground state $\mathrm{Cl}$ is released in both steps of the decay with an averaged $\beta$ parameter of $0.2 \pm 0.2$. In the first step $\mathrm{Cl}$ atoms with an anisotropy parameter of $0.4 \pm 0.2$ are ejected, while in the second step the $\mathrm{SOCl}$ intermediate undergoes an isotropic unimolecular decay into $\mathrm{SO}+\mathrm{Cl}$ with a $\beta$ parameter of close to 0 .

\section{Appendix - Calculation of kinetic speed distribution from TOF profiles}

Generally, the full three-dimensional velocity distribution $P(v, \theta, \phi)$ can be expressed in spherical coordinates. Then, the speed distribution $P(v)$, which can easily be converted into the kinetic energy distribution $P\left(E_{\text {kin }}\right) \propto P(v) / v$, is obtained from integrating the three-dimensional velocity distribution over the angular coordinates:

$$
P(v)=\iint v^{2} \sin \theta \mathrm{d} \theta \mathrm{d} \phi P(v, \theta, \phi)
$$

If in a photodissociation experiment the cylindrical symmetry around the polarisation vector of the dissociating laser is conserved, $P(v, \theta, \phi)$ is characterized by the (speed-dependent) anisotropy parameter $\beta$ according to eqn. (5).

On the other hand, according to eqn. (4), the TOF profile $P(t)$ is a direct measurement of the $P\left(v_{\mathrm{z}}\right)$ distribution of the $v_{z}$ component of the fragment velocity $\vec{v}$. Mathematically, it is a one-dimensional projection of the three-dimensional velocity distribution onto the spectrometer axis $z$ :

$$
\begin{aligned}
P\left(v_{z}\right)= & \int_{v_{z}}^{\infty} \mathrm{d} v \iint \mathrm{d} v_{y} \mathrm{~d} v_{x} P\left(v ; v_{x}, v_{y}, v_{z}\right) \\
& \times \delta\left(v-\sqrt{\left.v_{x}^{2}+v_{y}^{2}+v_{z}^{2}\right)}\right.
\end{aligned}
$$

where the $(x, y, z)$ Cartesian coordinate system is attached to the spectrometer axis $z$. The $\delta$ function only serves to satisfy the condition $v^{2}=v_{x}{ }^{2}+v_{y}{ }^{2}+v_{z}{ }^{2}$. Then, upon proper transformation of the coordinate systems, the experimentally observed TOF profile, represented by $\left(P\left(v_{z}\right)\right.$, and the desired kinetic energy distribution, represented by $P(v)$, are related by:

$$
P\left(v_{z}\right)=\int_{v_{z}}^{\infty} \mathrm{d} v\left(\frac{P(v)}{2 v}\left(1+\beta(v) P_{2}\left(\cos \frac{v_{z}}{v} P_{2}(\cos \gamma)\right)\right)\right.
$$

where $\gamma$ is the angle of the electric field vector of the dissociation laser with the spectrometer axis $z$. In general, this equation cannot analytically be inverted in order to obtain $P(v)$ from $P\left(v_{z}\right)$. Often, the relationship between $P(v)$ and $P\left(v_{z}\right)$ is even more complicated. Especially for a large kinetic energy release the laser bandwidth $\delta \nu_{\mathrm{L}}$ is usually smaller than the Doppler width $\Delta \nu_{\mathrm{D}}$ of the absorption lines of the fragments, and the selective excitation of a fragment subset with small velocity components $v_{x}$ along the laser beam axis must be considered. Then, the $v_{y}$ integration cannot be performed anymore, and eqn. (A3) becomes:

$$
\begin{aligned}
& P\left(v_{z}\right)=\int_{v_{z}}^{\infty} \mathrm{d} v \frac{1}{2 \pi v} \int_{-\sqrt{v^{2}-v_{z}^{2}}}^{\sqrt{v^{2}-v_{z}^{2}}} \mathrm{~d} v_{y} h\left(v, v_{y}, v_{z}\right) \\
& \text { with } h\left(v, v_{y}, v_{z}\right)= \\
& \frac{P(v) L\left(v, v_{y}, v_{z}\right)}{\sqrt{v^{2}-v_{y}^{2}-v_{z}^{2}}} \\
& \times\left(1+\beta(v) P_{2}\left(\frac{v_{y} \sin \gamma+v_{2} \cos \gamma}{v}\right)\right)
\end{aligned}
$$

$L$ is the normalised excitation probability function taking into account the finite laser bandwidth $\delta \nu_{\mathrm{L}}$. For a Gaussian laser intensity distribution around the centre $\nu_{0}$ of the transition $L \propto \exp \left(-\Delta \nu^{2} / 2 \delta \nu_{\mathrm{L}}^{2}\right)$ where $\Delta \nu$ is the deviation of the photolysis laser frequency $\nu$ within the laser bandwidth from $\nu_{0}$. In terms of velocity components $L \propto \exp \left(-\nu_{0}^{2} v_{\mathrm{x}}^{2} / 2 c^{2} \delta \nu_{\mathrm{L}}^{2}\right)=$ $\exp \left(v_{y}{ }^{2}+v_{z}{ }^{2}-v^{2} / a^{2}\right)$ where $a^{2}=\left(\nu_{0}^{2} / 2 c^{2} \delta \nu_{\mathrm{L}}{ }^{2}\right)$.

For a least squares fit a function $P_{\mathrm{F}}\left(v_{z}\right)$ is defined where the desired distribution function $P(v)$ is approximated by $P_{\mathrm{F}}(v)$, a sum over $n_{\max }$ rectangular functions $R_{n}(v)$ of equal width $v_{\max } /$ $n_{\max }$ with amplitude $b_{n}: P_{\mathrm{F}}(v)=\Sigma b_{n} R_{n}(v)$. Here $R_{n}(v)=1$ if $n / n_{\max }<v / v_{\max } \leqslant(n+1) / n_{\max }$ and $R_{n}(v)=0$ otherwise. $v_{\max }$ is the maximum available speed agreeing with energy conservation. The $b_{n}$ are adjusted such that the error sum $Q=$ $\sum\left(P_{\mathrm{F}}\left(v_{z}\right)-P_{\exp }\left(v_{z}\right)\right)^{2}$ is minimized, simultaneously satisfying the conditions $\partial Q / \partial b_{n}=0$ for all $n$. Here the summation is performed over all $v_{z}$ values and $P_{\exp }\left(v_{z}\right)$ is the experimentally 
observed distribution (i.e. the TOF profile scaled according to eqn. (4)). The $n_{\max }$ conditions form a linear equation system that can be solved analytically.

In this work a speed dependent $\beta$ parameter was considered, in that $\beta$ was approximated by a series of $\beta_{n}$ values, but $\beta$ was not explicitly included in the fitting procedure. This is possible, however, if $\beta(v)$ were approximated by a second order polynom, resulting in a coupled linear equation system. All calculations have been performed for $n_{\max }=20$.

\section{Acknowledgements}

Financial support from the Deutsche Forschungsgemeinschaft and the German-Israeli Foundation are gratefully acknowledged. M.R. thanks the Fonds der Chemischen Industrie for a fellowship.

\section{References}

1 J. H. D. Eland, Meas. Sci. Technol., 1994, 5, 1501.

2 O. Jagutzki, V. Mergel, K. Ullmann-Pfleger, L. Spielberger, U. Meyer, R. Dörner and H. Schmidt-Böcking, in Imaging Spectrometry IV, eds. M. R. Descour and S. S. Shen, Proc. SPIE, 1998, $3438,322$.

3 C. Maul and K.-H. Gericke, Int. Rev. Phys. Chem., 1997, 16, 1.

4 C. Maul and K.-H. Gericke, J. Phys. Chem. A, 2000, 104, 2531.

5 R. E. Continetti, Annu. Rev. Phys. Chem., 2001, 52, 165.

6 S. Kim, S. Pedersen and A. H. Zewail, J. Chem. Phys., 1995, 103 1006.

7 S. W. North, D. A. Blank, J. D. Gezelter, C. A. Longfellow and Y. T. Lee, J. Chem. Phys., 1995, 102, 4447.

8 G. E. Hall, D. VandenBout and T. J. Sears, J. Chem. Phys., 1991, 94, 4182.

9 K. A. Trentelman, S. H. Kable, D. B. Moss and P. L. Houston, J. Chem. Phys., 1989, 91, 7498.

10 P. M. Kroger and S. J. Riley, J. Chem. Phys., 1977, 67, 4483.

11 P. M. Kroger and S. J. Riley, J. Chem. Phys., 1979, 70, 3863

12 S. Deshmukh and W. P. Hess, J. Chem. Phys., 1994, 100, 6429.

13 S. Deshmukh, J. D. Myers, S. S. Xanthaes and W. P. Hess, J. Phys. Chem., 1994, 98, 12535.

14 I. C. Lane, R. Meehan and I. Powis, J. Phys. Chem., 1995, 99, 12371 .

15 C. Maul, T. Haas and K.-H. Gericke, J. Phys. Chem. A, 1997, 101, 6619.

16 C. Maul, T. Haas, K.-H. Gericke and F. J. Comes, J. Chem. Phys., 1995, 102, 3238.

17 T. Einfeld, A. Chichinin, C. Maul and K.-H. Gericke, J. Chem. Phys., 2002, 116, 2803.
18 C. Maul, C. Dietrich, T. Haas and K.-H. Gericke, Phys. Chem. Chem. Phys., 1999, 1, 1441.

19 X. Chen, H. Wang, B. R. Weiner, M. Hawley and H. H. Nelson, J. Phys. Chem., 1993, 97, 12269.

20 X. Chen, F. Asmar, H. Wang and B. R. Weiner, J. Phys. Chem., 1991, 95, 6415

21 H. Wang, X. Chen and B. R. Weiner, J. Phys. Chem., 1993, 97, 12260 .

22 G. Baum, C. S. Effenhauser, P. Felder and J. R. Huber, J. Phys. Chem., 1992, 96, 756.

23 G. Baum, Ph.D. Thesis, Universität Zürich, 1993.

24 M. Kawasaki, K. Kasatani, H. Sato, H. Shinohara, N. Nishi, H. Ohtoshi and I. Tanaka, Chem. Phys., 1984, 91, 285.

25 M. Roth, Untersuchung photoinduzierter Dreikörperzerfälle am Beispiel von $\mathrm{Cl}_{2} \mathrm{O}$ und $\mathrm{SOCl}_{2}$, dissertation.de, Berlin, Germany, 2001, (http://www.dissertation.de/PDF/mr418.pdf).

26 A. P. Uthman, P. J. Demlein, T. D. Aliston, M. C. Withlam, M. J. McClements and G. A. Takacs, J. Phys. Chem., 1978, 82, 2252.

27 T. Yamabe, S. Nagata, Y. Kikukzono and K. Fukui, Bull. Chem. Soc. Jpn., 1975, 48, 1349.

28 M. Kawasaki, K. Suto, Y. Sato, Y. Matsumi and R. Bersohn, J. Phys. Chem., 1996, 100, 19853.

29 M. Bogey, S. Civiš, B. Delcroix, C. Demuynck, A. F. Krupnov, J. Quiguer, M. Y. Tretyakov and A. Walters, J. Mol. Spectrosc., 1997, 182, 85 .

30 T. Haas, C. Maul, K.-H. Gericke and F. J. Comes, Chem. Phys. Lett., 1993, 202, 108.

31 C. P. Archer, J. M. F. Elks and C. M. Western, J. Chem. Phys., 2000, 112, 6293.

32 G. Huber and G. Herzberg, Constants of Diatomic Molecules, Molecular Spectra and Molecular Structure IV, Van Nostrand Reinhold, New York,1979.

33 S. Arepalli, N. Presser, D. Robie and R. Gordon, Chem. Phys. Lett., 1985, 118, 88.

34 D. Ascenzi, P. M. Regan and A. J. Orr-Ewing, Chem. Phys. Lett. 1999, 310, 477.

35 Y. Matsumi, K. Tonokura and M. Kawasaki, J. Chem. Phys, 1992, 97, 1065.

36 L. Li, R. J. Lipert, J. Lobue, W. A. Chupka and S. D. Colson, Chem. Phys. Lett., 1988, 151, 335.

37 C. Braatz and E. Tiemann, Chem. Phys., 1998, 229, 93.

38 C. Braatz, Ph.D. Thesis, Universität Hannover, 1997.

39 P. M. Regan, S. R. Langford, D. Ascenzi, P. A. Cook, A. J. OrrEwing and M. N. R. Ashfold, Phys. Chem. Chem. Phys., 1999, 1, 3247 .

40 I. Kovács, Rotational Structure in the Spectra of Diatomic Molecules, Adam Hilger, London, 1969.

41 X. Chen, I. Bar, S. Rosenwaks, T. Einfeld, C. Maul and K.-H. Gericke, J. Chem. Phys., 2001, 114, 9033.

42 R. S. Speth, C. Braatz and E. Tiemann, J. Mol. Spectrosc., 1998, 192, 69.

43 Z. Li, J. Phys. Chem. A, 1997, 101, 9545.

44 M. Roth, C. Maul and K.-H. Gericke, J. Chem. Phys., 1997, 107, 10582 .

45 F. Mata and N. Carballo, J. Mol. Struct., 1983, 101, 233. 